\title{
Oncologist
}

\section{Current Approaches to the Treatment of Early Hepatocellular Carcinoma}

\author{
Sheng-Long Ye, ${ }^{a}$ Tadatoshi Takayama, ${ }^{\text {b JefF Geschwind, }},{ }^{\text {J Jorge A. Marrero, }}{ }^{\text {d }}$ \\ JEAN-PIERRE BRONOWICKI ${ }^{\mathrm{e}}$
}

\begin{abstract}
${ }^{\text {a}}$ Liver Cancer Institute and Zhongshan Hospital, Fudan University, Shanghai, China; ${ }^{\mathrm{b}}$ Department of
Digestive Surgery, Nihon University School of Medicine, Tokyo, Japan; ${ }^{\mathrm{c}}$ Vascular and Interventional Radiology, Johns Hopkins University School of Medicine, Baltimore, Maryland, USA; ${ }^{\mathrm{d}}$ Multidisciplinary

Liver Tumor Program, University of Michigan, Ann Arbor, Michigan, USA; ${ }^{\circ}$ Department of Gastroenterology and Hepatology, INSERM U954, University Hospital of Nancy, University Henri Poincaré,

Vandœuvre-lès-Nancy, France
\end{abstract}

Key Words. Hepatocellular carcinoma • Liver transplantation • Ablation techniques • Operative surgical procedure

Disclosures: Sheng-Long Ye: None; Tadatoshi Takayama: None; Jeff Geschwind: Consultant/advisory role: Biosphere Medical, Biocompatibles, Bayer HealthCare, Guerbet; Research funding/contracted research: Biosphere Medical, Biocompatibles, Genentech, Bayer HealthCare, Philips Medical, Context Vision, Gideon, RSNA; Jorge A. Marrero: Honoraria: Bayer, Onyx; Research funding/contracted research: Bayer; Jean-Pierre Bronowicki: Honoraria: Bayer Pharma, Bristol-Myers Squibb.

The content of this article has been reviewed by independent peer reviewers to ensure that it is balanced, objective, and free from commercial bias. No financial relationships relevant to the content of this article have been disclosed by the independent peer reviewers.

\begin{abstract}
For patients with early-stage hepatocellular carcinoma (HCC), potentially curative treatment options exist, including liver transplantation, surgical resection, and ablation therapy. These treatments are associated with survival benefits, and outcomes are optimized by identification of appropriate patients. However, further studies are needed to definitively confirm optimal treatment approaches for all patients.

Treatment patterns vary in different parts of the world as a result of geographic differences in the incidence and presentation of the disease. In particular, because of successful screening programs, a high proportion of tumors that are identified in Japan are amenable to curative treatments, which are appropriate in a
\end{abstract}

smaller proportion of patients in the west, although screening is now widely carried out in industrialized countries. Differences in the applicability of transplantation are also evident between the west and Asia.

Although existing treatments for early-stage $\mathrm{HCC}$ are supported by considerable evidence, there remain significant data gaps. For example, further data, ideally from randomized controlled trials, are needed regarding: the use of neoadjuvant and adjuvant therapy to decrease the rate of recurrence after resection or ablation, further investigation of the role of chemoprevention following resection, and prospective analysis of outcomes of living donor compared with deceased donor liver transplantation. The Oncologist 2010;15(suppl 4):34-41 


\section{INTRODUCTION}

Hepatocellular carcinoma (HCC) is an increasingly prevalent clinical problem worldwide and is the third most common cause of cancer-related death $[1,2]$. The presence of cirrhosis is a key risk factor [3]. HCC is a complex disease involving many factors, and HCC staging systems can be very complicated [4]. The widely used, comprehensive Barcelona Clinic Liver Cancer staging system takes into account variables related to tumor stage, liver function, physical status, and cancer-related symptoms to generate a treatment algorithm [5].

Treatment is most effective in the early stages of disease, but diagnosing early-stage HCC is difficult because the diagnosis of cirrhosis is often not made before the emergence of HCC. Patients at high risk for developing HCC (e.g., those with cirrhosis, hepatitis B virus, or hepatitis C virus) should be entered into surveillance programs using ultrasound and serum $\alpha$-fetoprotein (AFP) [3, 6, 7]. Based mainly on observational data on tumor-volume doubling time, a screening interval of 6 months is commonly used by physicians in the West, in contrast to the Far East, where a 3-month screening interval is generally implemented [8]. In a recent meta-analysis, a significantly higher sensitivity for early HCC was observed with a 6-month interval than with annual surveillance [9]. Because of the high rates of falsepositive and false-negative results in patients with chronic liver disease, the American Association for the Study of Liver Diseases (AASLD) does not recommend the use of AFP alone as a screening method, unless ultrasound is not available. Information from a recent meta-analysis demonstrated that AFP provided no additional benefit to ultrasound, further supporting this guidance [9]. In contrast, abdominal ultrasonography combined with measurements of tumor markers is recommended for HCC screening, and assessments of AFP, protein induced by vitamin $\mathrm{K}$ absence or antagonist-II, or AFP lectin fraction are routinely performed in Japan [10].

Individuals with abnormal screening results require further investigation (e.g., with computed tomography scanning, magnetic resonance imaging, or liver biopsy) to confirm a diagnosis of HCC. Although surveillance programs can lead to detection of HCC at early stages when the tumors are amenable to curative treatment, guidelines are not always followed and are not always reproducible from large hospitals to nontertiary hospitals. Further studies are warranted to determine the optimal surveillance methods, which may also involve evaluation of novel biomarkers in the future.

Treatments for early-stage HCC include hepatectomy, liver transplantation, and local ablation therapy (Fig. 1) [6, 10-13]. However, there are no large randomized controlled trials (RCTs) comparing these treatments directly, nor are there any studies comparing these treatments with best supportive care [6]. In an intent-to-treat analysis in cirrhotic patients with HCC, early findings suggested similar survival rates in a comparison of resection with transplantation [14]. However, patient dropouts from waiting lists significantly impacted the longer-term findings in the transplantation group, and the authors concluded that resection may provide a better outcome for properly selected candidates. Further research is needed to confirm the optimal strategy based on the currently available treatments, and careful selection of patients is important in all approaches. Applicability of these treatments varies according to geographic distribution, with 50\%-70\% of cases in Japan (where there is widespread surveillance and a broad application of treatments) being suitable for curative treatment, compared with $25 \%-40 \%$ of cases in Europe and the U.S., and $<10 \%$ in Africa [15]. Data from a nationwide survey in Japan indicate that a single early HCC patient has a high chance of prolonged survival with resection, ablation, or transplantation [16]. The aim of this article is to review the therapeutic options and associated outcomes for the management of patients with early HCC.

\section{OUTCOMES AND TOLERABILITY OF EARLY-STAGE HCC TREATMENTS}

\section{Resection}

Patients with early-stage HCC are those most likely to benefit from curative interventions. In a study of patients diagnosed with HCC in 1988-1998 in the Surveillance, Epidemiology, and End Results database, 417 of the 4,008 patients were candidates for surgical resection. The study showed that surgery was associated with longer survival in patients with unifocal, nonmetastatic $\mathrm{HCC}$ tumors $<5 \mathrm{~cm}$. In patients receiving surgery, the 5-year overall survival (OS) rate was $33 \%$, compared with $7 \%$ without surgery [17].

Surgical resection is recommended as treatment for early HCC in noncirrhotic patients, or in patients with cirrhosis who have a single lesion and well-preserved liver function, normal bilirubin, and no portal hypertension [6, 13]. However, there are data that suggest that portal hypertension may not necessarily be a contraindication for resection. Patients with the same model for end-stage liver disease score and extent of hepatectomy had similar outcomes, whether or not they had portal hypertension [18], whereas several other studies found that resection can be performed safely in selected patients even in the presence of portal hypertension $[19,20]$. Patients with multiple tumors may also be suitable for resection, although tumor multiplicity is an independent risk factor for postoperative recur- 


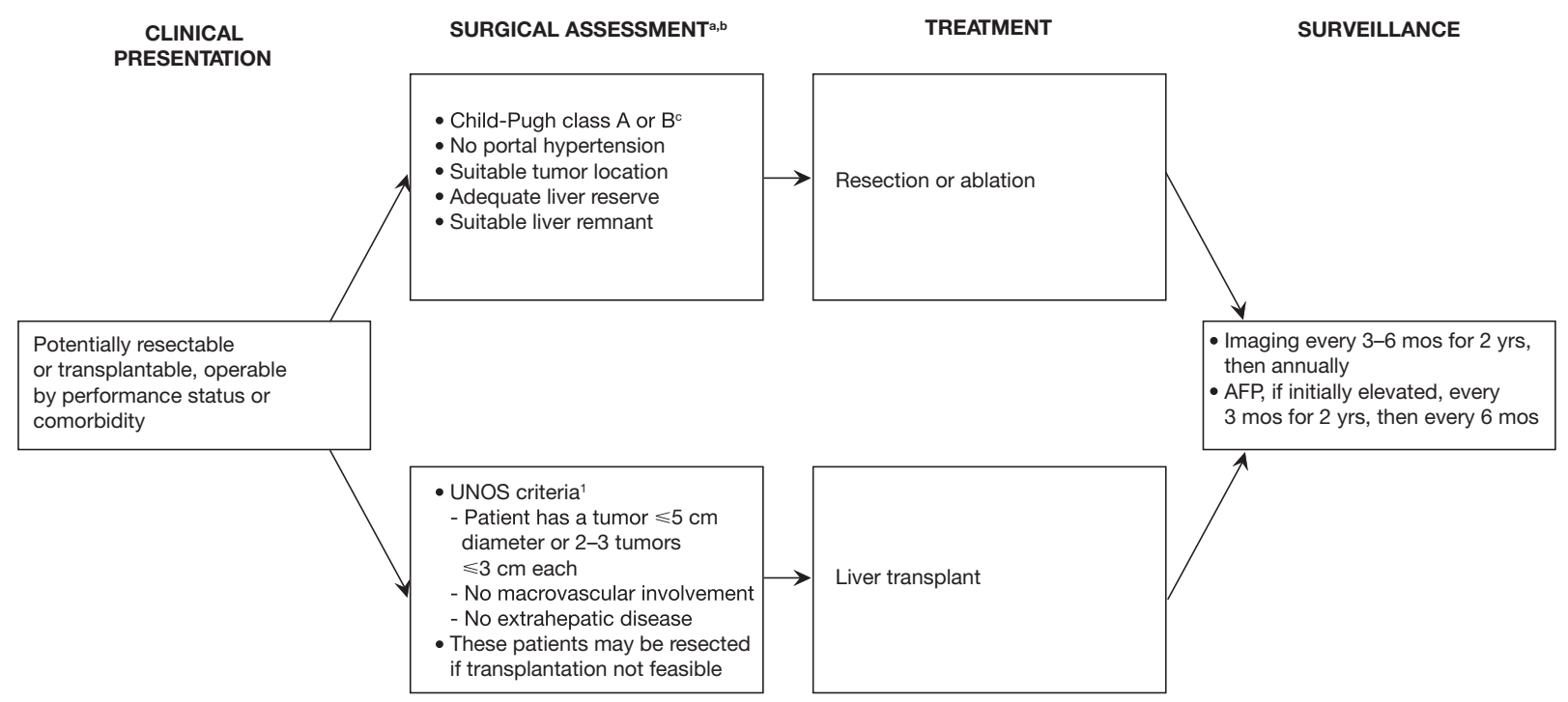

Figure 1. National Comprehensive Cancer Network guidelines for the treatment of potentially resectable disease.

${ }^{a}$ Discussion of surgical treatment with patient and determination of whether patient is amenable to surgery.

${ }^{\mathrm{b}}$ Patients with Child-Pugh class A liver function who fit UNOS criteria and are resectable could be considered for resection or transplant. There is controversy over which initial strategy is preferable to treat such patients. These patients should be evaluated by a multidisciplinary team.

${ }^{\mathrm{c}}$ In highly selected Child-Pugh class B patients with limited resection.

${ }^{1}$ Mazzaferro V, Regalia E, Doci R et al. Liver transplantation for the treatment of small hepatocellular carcinomas in patients with cirrhosis. N Engl J Med 1996;334:693-700.

Abbreviations: AFP, $\alpha$-fetoprotein; UNOS, United Network for Organ Sharing.

From National Comprehensive Cancer Network. NCCN Clinical Practice Guidelines in Oncology. Hepatobiliary Cancers V. 1.2010. Available at http://www.nccn.org/professionals/physician_gls/f_guidelines.asp. Reproduced with permission from The NCCN Clinical Practice Guidelines in Oncology ${ }^{\mathrm{TM}}$ Hepatobiliary Cancer Guidelines. (02009 National Comprehensive Cancer Network, Inc. All rights reserved. These Guidelines and illustrations herein may not be reproduced in any form for any purpose without the express written permission of the NCCN. To view the most recent and complete version of the NCCN Guidelines, go online to NCCN.

rence, and the OS time is shorter in these patients [20]. However, among patients with both multiple tumors and better liver function (Child-Pugh class A), an absolute 5 -year survival rate of $58 \%$ was achieved. Although there is no limitation on tumor size for resection, the risk for vascular invasion and dissemination increases with size. The amount of liver that can be resected depends on the degree of cirrhosis, the functional liver reserve, and the regenerative capacity of the liver [7]. Strict selection criteria are required in order to avoid treatment-related complications such as liver failure. Survival rates of $\sim 70 \%$ at 5 years have been achieved in patients with a normal bilirubin concentration and no clinically significant portal hypertension [6]. In Japan, the indocyanine green retention rate, a marker of hepatic clearance, is commonly used to predict the safe limit of liver resection and posthepatectomy liver failure [21]. Preoperative portal vein embolization (PVE) has been used to evaluate the regenerative abilities of the liver, with lack of hypertrophy following PVE indicating an inability of the liver to regenerate, therefore contraindicating major liver resection [22]. Furthermore, preoperative PVE has been shown to improve outcomes following major hepatectomy [23]. In patients with very early HCC (carcinoma in situ) undergoing surgery, the best 5-year survival rate so far, 93\%, was demonstrated [24]. Only 10\%-30\% of HCC cases are suitable for "curative" surgical resection at the time of diagnosis, and recurrent $\mathrm{HCC}$ has been reported in $50 \%-80 \%$ of patients 5 years after resection [7]. Key predictors of recurrence are the presence of microvascular invasion and/or further tumor sites in addition to the primary lesion. Preoperative transcatheter arterial chemoembolization (TACE) has been evaluated but has shown no benefit in this setting [7]. AASLD and Japanese guidelines conclude that there is currently no preoperative or postoperative adjuvant therapy that can be recommended for improving prognosis after hepatic resection $[6,10]$. Further investigation is required for neoadjuvant and adjuvant therapies that may decrease the incidence of recurrence following resection.

\section{Transplantation}

Liver transplantation as a treatment for early-stage HCC is well established in the U.S. and Europe and is associated 
with 5-year survival rates of $\sim 70 \%$ [6], comparable with those of noncancer liver recipients. In most centers, candidates for transplantation are deemed not resectable. In some parts of the world, transplantation is not available or has very limited applicability [6]. The benefits of liver transplantation over resection include removal of the tumor and the underlying diseased liver and also improvement in portal hypertension. Because of the limited supply of donor organs, identification of the patients most likely to receive maximum benefit from a transplant is of utmost importance. For over a decade, the Milan criteria for HCC (one lesion $\leq 5 \mathrm{~cm}$ or two to three lesions $\leq 3 \mathrm{~cm}$ ) have been widely used for the selection of candidates for liver transplantation. However, there is an ongoing debate on whether expanded criteria may be adopted, to enable patients with slightly more advanced HCC to also benefit from liver transplantation [25]. A 5-year survival rate of $\sim 50 \%$ was described in patients selected with such expanded criteria, but there are currently no clear data to define the new limits [6]. In addition, expanding the criteria may cause harm to other patients without cancer who need a transplant, as a result of fewer donors being available [26]. Because the waiting time for an organ to become available may exceed 12 months in some western countries [27], the dropout rate is high (up to 50\%). Most centers administer adjuvant treatments to prevent tumor progression while patients are on the waiting list, but these are often chosen based on observational studies, because robust data from RCTs are not available. Such bridging therapy before transplantation may include locoregional therapy such as chemoembolization, which has been investigated as a means of downstaging tumors to facilitate liver transplantation [25]. Information from a liver transplant waiting list in the U.S. showed that HCC patients who received pretransplant ablation treatments had a higher adjusted 3-year post-transplant survival rate than HCC transplant patients who did not (79\% versus $75 \% ; p=.03$ ) [28]. However, in another retrospective cohort study in the U.S., using data from a liver transplant waiting list, the authors concluded that the effects of downstaging with neoadjuvant treatment were difficult to evaluate [29]. It has also been suggested that resection can be used as a bridging therapy for patients who have already been enlisted for liver transplant [30]. There is no definitive evidence confirming that the use of bridging therapies confers an advantage post-transplantation in terms of survival and recurrence rates, and no specific recommendations in relation to bridging strategies (for either TACE or local ablation therapy) are currently made in the guidelines [7, 13].

An alternative strategy to increase the pool of available donor livers is the use of live donor transplantation, which

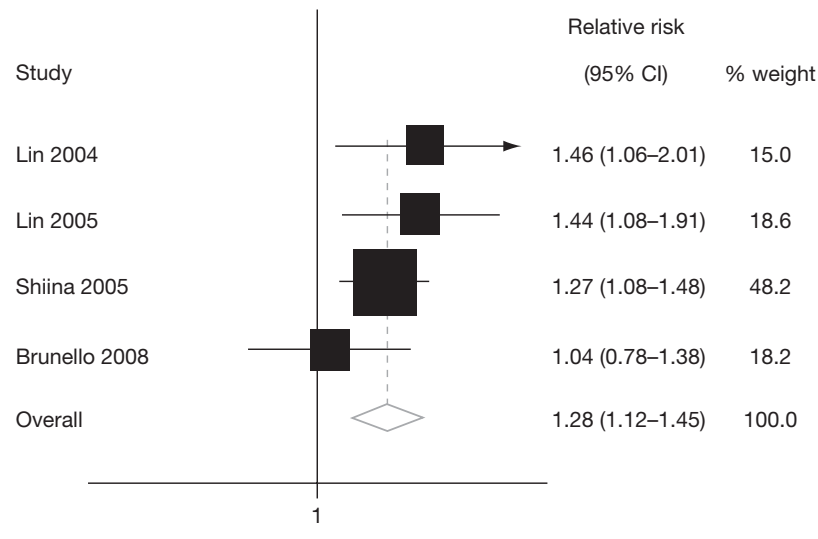

Figure 2. Radiofrequency ablation versus percutaneous ethanol injection: results of the meta-analysis on overall survival at 3 years. All based on random-effects meta-analysis.

Abbreviation: CI, confidence interval.

From Bouza C, López-Cuadrado T, Alcázar R et al. Metaanalysis of percutaneous radiofrequency ablation versus ethanol injection in hepatocellular carcinoma. BMC Gastroenterol 2009;9:31, with permission. Originally published by BioMed Central.

originated in Asia as a result of the legal and societal constraints on cadaveric liver transplantation [27, 31]. The results appear to be comparable with those from cadaveric donation [7, 32]; however, this is a complex intervention and may not have wide applicability.

\section{Ablation}

Local ablation therapy, with either radiofrequency ablation (RFA) or percutaneous ethanol injection (PEI), is commonly used to treat small HCCs confined to the liver that may be unresectable because of the poor general condition or compromised liver function of the patient. In an RCT comparing RFA with PEI for early HCC, the 1-year complete response rate was better with RFA than with PEI, although no clear survival advantage was observed in cirrhotic patients [33]. However, other RCTs [34-36] and a recent meta-analysis [37] have shown evidence of the superiority of RFA over PEI, in terms of longer survival and better local control of disease, in patients with relatively preserved liver function and early-stage nonsurgical HCC (Fig. 2). At 3 years, the pooled analysis showed an OS rate of $73 \%$ in the RFA group, compared with $58 \%$ in the PEI group $(p<.001)$ [37]. However, RFA was associated with a statistically significant higher rate of adverse events $(p<$ .001 ), with $19 \%$ of patients ( $95 \%$ confidence interval [CI], $15 \%-23 \%$ ) experiencing complications, compared with $10.5 \%$ of those treated with PEI (95\% CI, 7\%-13.5\%) [37]. The most frequent complication observed in that study was severe pain, which was more common with RFA than with PEI [37]. For studies that reported major complications, the 
incidence in RFA-treated patients was $4.1 \%$ (95\% CI, $1.8 \%-6.4 \%$ ), including hemothorax requiring thoracostomy drainage, gastric bleeding, hemoperitoneum, transitory icterus, liver infarction, cutaneous burn, and tumoral cell seeding, and in PEI-treated patients it was 2.7\% (95\% CI, $0.4 \%-5.1 \%$ ), including liver abscess, hemoperitoneum, tumoral cell seeding, and one procedure-related death; however, this difference was not statistically significant. This safety profile should be taken into consideration as part of the overall risk-benefit profile in each individual case. Further support for the benefit of RFA was provided by a different meta-analysis, which was more selective in the studies that it included and showed a higher 3-year OS rate with RFA than with PEI (odds ratio, 0.47; 95\% CI, $0.340-0.670 ; p<.001)$ in patients with small HCCs [38].

Local ablation therapy has been compared with resection in a number of retrospective studies and clinical trials. Long-term outcomes in 87 patients with single-nodule HCCs treated with either surgical resection or RFA were similar [39]. Similarly, 5-year survival rates were comparable in a study of 224 patients with Child-Pugh class A cirrhosis treated with either resection $(70.4 \%)$ or RFA $(76.8 \%)$ $(p=.561)$ [40]. A study of 186 patients with small $(<5 \mathrm{~cm})$ HCCs found that the choice of treatment should be based on local factors, such as the availability of resources and expertise [41]. In contrast to these findings, a study of 149 patients with $\mathrm{HCCs} \leq 4 \mathrm{~cm}$ comparing resection with percutaneous ablation found that resection provided better local control and better long-term survival (median survival time, 122 months after hepatectomy compared with 66 months after ablation; $p=.0123$ ) [42]. A nationwide survey in Japan generated data on survival following resection or RFA [16]. In 2000-2003, 1,235 patients with a single early $\mathrm{HCC}(<2 \mathrm{~cm})$ underwent resection and 1,315 patients received RFA. Although, with a median follow-up of 37 months, the disease-free survival rate was significantly better after resection than after RFA ( 1 year, $91 \%$ versus $84 \%$; 2 years, $70 \%$ versus $58 \%$; $p=.001$ ), there was no significant difference in the OS rate between the two groups (98\% versus $99 \%$; $94 \%$ versus $95 \% ; p=.28$ ). However, it is currently unknown whether the better disease-free survival seen with resection will translate into longer survival over a longer time period following therapy. Local ablation therapy was compared with resection in two RCTs in patients with small HCCs, with comparable survival results [43, 44]. Based on a trial of 180 patients, Chen et al. [43] concluded that RFA was as effective as surgical resection in the treatment of solitary and small HCCs, with the advantage of being less invasive. In a smaller study of 76 patients, Huang et al. [44] reported that PEI appeared to be as safe and effective as resection. Recent studies have shown that, in some centers, RFA is regarded as the first-line treatment for small, operable HCCs $(\leq 2 \mathrm{~cm})$, with $68.5 \%$ of patients surviving at 5 years [45]. Furthermore, in a simulated randomized trial comparing hepatic resection with RFA for very early HCCs $(<2 \mathrm{~cm})$, the OS times were similar for resection and RFA followed by resection for cases of initial local failure, suggesting that RFA could be considered as a primary treatment for very early HCC [46]. Given these equivocal results, larger RCTs are needed before there is any change in the recommended treatment of patients with good surgical risk and before ablation therapy is confirmed as an alternative to surgery for potentially resectable HCC.

\section{TACE}

Embolization procedures are used in patients with inoperable or unresectable disease. However, the place of TACE for the treatment of early HCC is not clear, and official guidelines do not currently recommend it. Caution should be exercised regarding the use of TACE for early HCC, and it should be considered only when curative treatment (e.g., transplantation, resection, or RFA) is contraindicated.

\section{Differences in the Treatment of EARLy HCC and Outcomes Between Populations}

As described above, well-defined treatment options for early HCC exist; however, there are inevitable differences in the treatment received, and hence the outcome achieved, in different populations worldwide. There are geographic variations in the incidence and etiology of $\mathrm{HCC}$, and a difference in tumor size at presentation. Japanese patients have been shown to present with smaller tumors than patients in the U.S. and Europe, likely as a result of the more widespread screening carried out in Japan [47]. This, together with differences in hepatitis $\mathrm{B}$ or $\mathrm{C}$ virus status, has resulted in more limited surgical resections being necessary in Japan, compared with more extended resections in the U.S.

In a more recent comparison, analysis of the medical records of 353 patients subject to surgical resection for $\mathrm{HCC}$ at two referral centers in China and Japan highlighted differences between populations [48]. As well as demographic differences in age of incidence, serum examination, and history of viral infection, differences in outcome were observed. Patients in Japan were diagnosed earlier, were subject to more standard treatment, and had better prognoses than those in China. However, these results were based only on HCC at each center and not on HCC detected in a surveillance program. In addition, the demographic disparities in survival in patients with localized HCC in the U.S. were investigated in a retrospective cohort study using data from the Surveillance, Epidemiology, and End Results 
population-based cancer registry [49]. That study found substantial and significant disparities by race/ethnicity in the 3-year survival rate, therapy administered, and stagespecific survival rate for individual therapies. These differences were not explained by age, date of diagnosis, or geography, but may have resulted from differences in treatments received by different demographic groups or variations in treatment response, which may be influenced by compliance or differences in disease biology. However, these patients were not identified through a surveillance program, but were patients diagnosed with HCC, which may be associated with lead-time bias. In a prospective cohort study in Europe, hepatic resection performed under strict intraoperative ultrasonographic guidance had low mortality and acceptable morbidity, even in patients with intermediate and advanced HCC [50].

\section{IMPROVING TREATMENT OPTIONS}

There remains a considerable number of unanswered questions in the recommendations for treatment of early-stage HCC, many of which require a definitive answer to be provided through robust data from RCTs. Key areas for consideration include: the use of neoadjuvant or adjuvant therapy to decrease or delay recurrence after resection or ablation, chemoprevention after resection or ablation, and the use of molecular profiling of HCC to provide additional tools to define those patients most at risk for recurrence following resection. Indeed, a number of clinical trials are ongoing in these areas. Three ongoing phase IV trials are investigating radiotherapy (ClinicalTrials.gov identifier, NCT00557024), TACE (ClinicalTrials.gov identifier, NCT00556803), and lamivudine or entecavir (ClinicalTrials.gov identifier, NCT00555334) as adjuvant therapies after RFA, and are due to complete in 2010. Furthermore, sorafenib (Nexavar ${ }^{\circledR}$; Onyx Pharmaceuticals, Inc., Emeryville, CA; Bayer HealthCare Pharmaceuticals, Inc., Wayne, NJ; Bayer Schering Pharma AG, Berlin, Germany) is being investigated as adjuvant treatment in the prevention of recurrence of HCC following either surgical resection or local ablation, in the large phase III randomized, doubleblind, placebo-controlled Sorafenib as Adjuvant Treatment in the Prevention of Recurrence of Hepatocellular Carci- noma (STORM) trial (ClinicalTrials.gov identifier, NCT00692770), due to complete in 2011. With regard to liver transplantation, bridging therapy before transplantation, including the questions of which treatment to give and when [7], and prospective analysis of outcomes of living donor compared with deceased donor transplantation are areas that warrant further study.

Important considerations in future trials include analysis of the cost-effectiveness of the treatments under investigation and also the use of genomics- and proteomicsbased technologies [51], in order to add to the body of information on the biologic behavior and natural history of HCC, which should help guide the diagnosis and management of HCC.

\section{CONCLUSIONS}

Early diagnosis remains a key goal in order to improve the prognosis of HCC patients. Surgical resection and liver transplantation are usually considered as first-line options because they offer the possibility of prolonged survival in patients with early disease and have excellent outcomes in well-selected patients. Local ablation therapy, using RFA or PEI, also has a role to play. Further improvements in the outcome of patients with early HCC may be achieved once outstanding questions have been answered by prospective RCTs.

\section{ACKNOWLEDGMENTS}

The authors take full responsibility for the scope, direction, and content of the manuscript and have approved the submitted manuscript. They would like to thank Karen Brayshaw, $\mathrm{PhD}$, at Complete HealthVizion for her assistance in the preparation and revision of the draft manuscript, based on detailed discussion and feedback from all the authors. Editorial assistance was supported by a grant from Bayer HealthCare Pharmaceuticals.

\section{Author Contributions}

Conception/Design: Sheng-Long Ye, Tadatoshi Takayama, Jeff Geschwind, Jorge A. Marrero, Jean-Pierre Bronowicki

Data analysis and interpretation: Sheng-Long Ye, Tadatoshi Takayama, Jeff Geschwind, Jorge A. Marrero, Jean-Pierre Bronowicki

Manuscript writing: Sheng-Long Ye, Tadatoshi Takayama, Jeff Geschwind, Jorge A. Marrero, Jean-Pierre Bronowicki

Final approval of manuscript: Sheng-Long Ye, Tadatoshi Takayama, Jeff Geschwind, Jorge A. Marrero, Jean-Pierre Bronowicki

\section{REFERENCES}

1 Parkin DM, Bray F, Ferlay J et al. Global cancer statistics, 2002. CA Cancer J Clin 2005;55:74-108.

2 Venook et al. The incidence and epidemiology of hepatocellular carcinoma: A global and regional perspective. The Oncologist 2010;15(suppl 4):5-13.

3 Sanyal et al. The etiology of hepatocellular carcinoma and consequences for treatment. The Oncologist 2010;15(suppl 4):14-22.
4 Marrero JA, Kudo M, Bronowicki JP. The challenge of prognosis and staging for hepatocellular carcinoma. The Oncologist 2010;15(suppl 4): 23-33.

5 Lencioni R, Chen XP, Dagher L et al. Treatment of intermediate/advanced hepatocellular carcinoma in the clinic: How can outcomes be improved? The Oncologist 2010;15(suppl 4):42-52.

6 Bruix J, Sherman M; Practice Guidelines Committee, American Associa- 
tion for the Study of Liver Diseases. Management of hepatocellular carcinoma. Hepatology 2005;42:1208-1236.

7 Lau WY, Lai ECH. Hepatocellular carcinoma: Current management and recent advances. Hepatobiliary Pancreat Dis Int 2008;7:237-257.

8 Colombo M. Screening and diagnosis of hepatocellular carcinoma. Liver Int 2009;29(suppl 1):143-147.

9 Singal A, Volk ML, Waljee A et al. Meta-analysis: Surveillance with ultrasound for early-stage hepatocellular carcinoma in patients with cirrhosis. Aliment Pharmacol Ther 2009;30:37-47.

10 Makuuchi M, Kokudo N, Arii S et al. Development of evidence-based clinical guidelines for the diagnosis and treatment of hepatocellular carcinoma in Japan. Hepatol Res 2008;38:37-51.

11 Llovet JM, Di Bisceglie AM, Bruix J et al. Design and endpoints of clinical trials in hepatocellular carcinoma. J Natl Cancer Inst 2008;100:698-711.

12 Ryder SD; British Society of Gastroenterology. Guidelines for the diagnosis and treatment of hepatocellular carcinoma (HCC) in adults. Gut 2003; 52(suppl 3):iii1-iii8.

13 National Comprehensive Cancer Network. NCCN Clinical Practice Guidelines in Oncology. Hepatobiliary Cancers V. 1.2010. Available at http:// www.nccn.org/professionals/physician_gls/f_guidelines.asp, accessed January 7, 2010.

14 Llovet JM, Fuster J, Bruix J. Intention-to-treat analysis of surgical treatment for early hepatocellular carcinoma: Resection versus transplantation. Hepatology 1999;30:1434-1440.

15 Llovet JM. Updated treatment approach to hepatocellular carcinoma. J Gastroenterol 2005;40:225-235.

16 Takayama T, Makuuchi M, Kojiro M et al. Early hepatocellular carcinoma: Pathology, imaging, and therapy. Ann Surg Oncol 2008;15:972-978.

17 Liu JH, Chen PW, Asch SM et al. Surgery for hepatocellular carcinoma: Does it improve survival? Ann Surg Oncol 2004;11:298-303.

18 Cucchetti A, Ercolani G, Vivarelli M et al. Is portal hypertension a contraindication to hepatic resection? Ann Surg 2009;250:922-928.

19 Capussotti L, Ferrero A, Viganò L et al. Portal hypertension: Contraindication to liver surgery? World J Surg 2006;30:992-999.

20 Ishizawa T, Hasegawa K, Aoki T et al. Neither multiple tumors nor portal hypertension are surgical contraindications for hepatocellular carcinoma. Gastroenterology 2008;134:1908-1916.

21 Nakayama H, Takayama T, Hemmi A. Postoperative complications of hepatectomy for hepatocellular carcinoma in relation to liver fibrosis. Hepatogastroenterology 2005;52:587-590.

22 Farges O, Belghiti J, Kianmanesh R et al. Portal vein embolization before right hepatectomy: Prospective clinical trial. Ann Surg 2003;237:208-217.

23 Palavecino M, Chun YS, Madoff DC et al. Major hepatic resection for hepatocellular carcinoma with or without portal vein embolization: Perioperative outcome and survival. Surgery 2009;145:399-405.

24 Takayama T, Makuuchi M, Hirohashi S et al. Early hepatocellular carcinoma as an entity with a high rate of surgical cure. Hepatology 1998;28: $1241-1246$.

25 Yao FY. Liver transplantation for hepatocellular carcinoma: Beyond the Milan criteria. Am J Transplant 2008;8:1982-1989.

26 Volk ML, Vijan S, Marrero JA. A novel model measuring the harm of transplanting hepatocellular carcinoma exceeding Milan criteria. Am J Transplant 2008;8:839-846.

27 Llovet JM, Burroughs A, Bruix J. Hepatocellular carcinoma. Lancet 2003; 362:1907-1917.

28 Freeman RB Jr, Steffick DE, Guidinger MK et al. Liver and intestine trans- plantation in the United States, 1997-2006. Am J Transplant 2008;8:958976.

29 Pelletier SJ, Fu S, Thyagarajan V et al. An intention-to-treat analysis of liver transplantation for hepatocellular carcinoma using organ procurement transplant network data. Liver Transpl 2009;15:859-868.

30 Belghiti J. Resection and liver transplantation for HCC. J Gastroenterol 2009;44(suppl 19):132-135.

$31 \mathrm{Ng} \mathrm{KK}$, Lo CM. Liver transplantation in Asia: Past, present and future. Ann Acad Med Singapore 2009;38:322-331.

32 Liu CL, Fan ST, Lo CM et al. Operative outcomes of adult-to-adult right lobe live donor liver transplantation: A comparative study with cadaveric whole-graft liver transplantation in a single center. Ann Surg 2006;243: $404-410$.

33 Brunello F, Veltri A, Carucci P et al. Radiofrequency ablation versus ethanol injection for early hepatocellular carcinoma: A randomized controlled trial. Scand J Gastroenterol 2008;43:727-735.

34 Lencioni RA, Allgaier HP, Cioni D et al. Small hepatocellular carcinoma in cirrhosis: Randomized comparison of radio-frequency thermal ablation versus percutaneous ethanol injection. Radiology 2003;228:235-240.

35 Lin SM, Lin CJ, Lin CC et al. Randomised controlled trial comparing percutaneous radiofrequency thermal ablation, percutaneous ethanol injection, and percutaneous acetic acid injection to treat hepatocellular carcinoma of $3 \mathrm{~cm}$ or less. Gut 2005;54:1151-1156.

36 Shiina S, Teratani T, Obi S et al. A randomized controlled trial of radiofrequency ablation with ethanol injection for small hepatocellular carcinoma. Gastroenterology 2005;129:122-130.

37 Bouza C, López-Cuadrado T, Alcázar R et al. Meta-analysis of percutaneous radiofrequency ablation versus ethanol injection in hepatocellular carcinoma. BMC Gastroenterol 2009;9:31.

38 Cho YK, Kim JK, Kim MY et al. Systematic review of randomized trials for hepatocellular carcinoma treated with percutaneous ablation therapies. Hepatology 2009;49:453-459.

39 Ogihara M, Wong LL, Machi J. Radiofrequency ablation versus surgical resection for single nodule hepatocellular carcinoma: Long-term outcomes. HPB (Oxford) 2005;7:214-221.

40 Takahashi S, Kudo M, Chung H et al. Outcomes of nontransplant potentially curative therapy for early-stage hepatocellular carcinoma in ChildPugh stage A cirrhosis is comparable with liver transplantation. Dig Dis 2007;25:303-309.

41 Farinati F, Gianni S, Marin G et al. Does the choice of treatment influence survival of patients with small hepatocellular carcinoma in compensated cirrhosis? Eur J Gastroenterol Hepatol 2001;13:1217-1224.

42 Wakai T, Shirai Y, Suda T et al. Long-term outcomes of hepatectomy vs percutaneous ablation for treatment of hepatocellular carcinoma $\leq 4 \mathrm{~cm}$. World J Gastroenterol 2006;12:546-552.

43 Chen MS, Li JQ, Zheng Y et al. A prospective randomized trial comparing percutaneous local ablative therapy and partial hepatectomy for small hepatocellular carcinoma. Ann Surg 2006;243:321-328.

44 Huang GT, Lee PH, Tsang YM et al. Percutaneous ethanol injection versus surgical resection for the treatment of small hepatocellular carcinoma: A prospective study. Ann Surg 2005;242:36-42.

45 Livraghi T, Meloni F, Di Stasi M et al. Sustained complete response and complications rates after radiofrequency ablation of very early hepatocellular carcinoma in cirrhosis: Is resection still the treatment of choice? Hepatology 2008;47:82-89.

46 Cho YK, Kim JK, Kim WT et al. Hepatic resection versus radiofrequency 
ablation for very early stage hepatocellular carcinoma: A Markov model analysis. Hepatology 2010;51:1284-1290.

47 Pawlik TM, Esnaola NF, Vauthey JN. Surgical treatment of hepatocellular carcinoma: Similar long-term results despite geographic variations. Liver Transpl 2004;10(suppl 1):S74-S80.

48 Yuan P, Tang W, Kokudo N et al. Demographic and clinical characteristics of resectable hepatocellular carcinoma in China and Japan. Hepatogastroenterology 2007;54:2343-2347.

49 Wong RJ, Corley DA. Survival differences by race/ethnicity and treatment for localized hepatocellular carcinoma within the United States. Dig Dis Sci 2009;54:2031-2039.

50 Torzilli G, Donadon M, Marconi M et al. Hepatectomy for stage B and stage $\mathrm{C}$ hepatocellular carcinoma in the Barcelona Clinic Liver Cancer classification: Results of a prospective analysis. Arch Surg 2008;143:10821090.

51 Pei Y, Zhang T, Renault V et al. An overview of hepatocellular carcinoma study by omics-based methods. Acta Biochim Biophys Sin (Shanghai) 2009;41:1-15. 